\title{
Um Sistema de Following para ampliar a interação em Comunidades de Prática
}

\author{
Matheus Pereira, João Luis Tavares da Silva e Elisa Boff \\ Universidade de Caxias do Sul (UCS) - RS \\ matheper@gmail.com,joaoluis.tavares@gmail.com,eboff@ucs.br
}

\begin{abstract}
Resumo. Este artigo apresenta um sistema de following, de usuários e temas, desenvolvido para ampliar e estimular a interação entre os participantes de comunidades de prática. Neste ambiente, conhecer e interagir com outros indivíduos é fundamental no processo de aprendizagem e a existência de ferramentas que apoiem as práticas é o que define o sucesso de uma comunidade. Esta rede de relacionamentos representa um interesse distribuído dos participantes por determinados assuntos. O sistema de following captura e representa estes interesses de modo a considerá-los para recomendações na plataforma CoPPLA. É por meio destas sugestões oferecidas pelo sistema de recomendação, que os usuários são convidados a colaborar com outros participantes ou a conhecer novos temas de interesse.
\end{abstract}

Palavras-chave: Colaboração, Comunidades de Prática, Sistemas de Recomendação, Ambientes de Aprendizagem

\section{A Following System to improve interaction in Communities of Practice}

\begin{abstract}
This paper presents a Following System, for users and topics, designed to improve and to encourage interaction among participants in communities of practice. In this environment, is very important to meet and to interact with other individuals in the learning process and also the supply of tools to support the practices are what define the success of a community. This relationships network can be compared with an interest of participants distributed by certain subjects. The Following System captures and represents these interests in order to consider them for recommendations on the Coppla platform. It is through these suggestions offered by the recommendation system, that users are invited to collaborate with other participants or to discover new topics of interest
\end{abstract}

Keywords: Collaboration, Communities of Practice, Recommendation Systems, Learning Environments

\section{Introdução}

Devido à rápida expansão e democratização da Internet, a produção de conteúdo segue em ritmo acelerado. Como resultado os usuários são expostos a uma grande oferta de informação (Zaiane, 2000) que dificulta a busca por conteúdos específicos e torna complexa a atividade de aprendizagem. 
O aspecto social da aprendizagem está relacionado à interação humana, ou seja, aprendizagem colaborativa através do contato e associações regulares com outros indivíduos. Bandura (1977) define social learning como um processo de observação do comportamento de outros indivíduos e suas consequências, para que o observador possa adaptar seu próprio comportamento de acordo com o grupo. Para que as CoP sejam bem sucedidas é necessário que elas sejam capazes de serem sistemas de aprendizagem social (Wenger, 2000). É neste contexto que Wenger identifica os elementos constitutivos desta aprendizagem social: Comunidades de Prática, Processos Limítrofes entre as comunidades e Identidades como forma de participação. Através da interação regular e da troca de experiências, as CoP podem suportar a aprendizagem social na medida em que seus membros aprendem sobre um domínio e também sobre a própria participação (Healy, 2009). Neste sentido, as CoP oferecem suporte à interação social ao mesmo tempo em que estimulam o processo de participação e aprofundamento do domínio de aprendizagem (RIBEIRO et al., 2011).

Este trabalho enquadra-se no contexto de aprendizagem colaborativa através da interação de usuários em comunidades virtuais. Em Fiorio (2011) e Ribeiro (2011) é proposto um framework de comunidades de prática (CoP) com o objetivo de oferecer uma plataforma de construção de comunidades virtuais voltadas ao desenvolvimento de práticas e habilidades relacionadas a um domínio específico. O principal foco desta abordagem é a modelagem de um framework que utiliza um modelo de referência ontológico para representar uma comunidade, seus participantes, o conhecimento e os perfis de interesse e domínio em um contexto de comunidades de prática.

A plataforma de comunidades de prática em questão, CoPPLA ${ }^{1}$ (2010), compreende um conjunto de ferramentas de comunicação e colaboração, integradas em um ambiente voltado para o compartilhamento de conhecimento, que envolve a manipulação de textos, imagens, páginas web, links, eventos, fóruns de discussão e espaços para experiências de aprendizagem. A plataforma faz com que a criação de conteúdo e a manipulação de objetos sejam flexíveis e intuitivas, promovendo maior participação dos usuários. A ampla atividade destes usuários gera uma grande quantidade de conteúdo que precisa ser acessado e encontrado por seus pares.

Uma das funcionalidades a ser desenvolvida é a possibilidade de conhecer e seguir (follow/following) outros participantes e temas. Temas são termos de categorização do conhecimento relacionados a cada conteúdo compartilhado nas comunidades. A possibilidade de seguir temas define uma parte dinâmica do interesse do usuário, que pode evoluir com as interações nas comunidades. Por outro lado, seguir pessoas é uma função bastante comum atualmente em redes sociais que relaciona o conteúdo postado por determinada pessoa aos interesses do seguidor, colocando este conteúdo a disposição de forma implícita. A principal forma de disponibilizar estes conteúdos e relacionamentos é através de recomendações. Com base neste cenário, o enfoque deste artigo é representar estes interesses, por temas e pessoas, através de uma estrutura que possa ser utilizada por um sistema de recomendação, baseado em filtragem colaborativa, que irá sugerir novos usuários, temas e comunidades no framework CoPPLA.

As próximas seções apresentam uma revisão bibliográfica dos conceitos referentes à tecnologia de comunidades de prática, sistemas de recomendações e a

\footnotetext{
${ }^{1}$ https://bitbucket.org/amribeiro/communities.practice
} 
plataforma de comunidades de prática CoPPLA, a proposta de solução para o sistema de following e as considerações finais.

\section{Tecnologias de Comunidades de Prática}

Comunidades de prática consistem em grupos de pessoas que compartilham determinado interesse comum. A aprendizagem e a produção do conhecimento ocorrem a partir da interação dos indivíduos (Wenger, 1998). Desta maneira, a comunidade de prática atribui ao aprendiz não somente o papel de consumidor, mas também de produtor da informação, desenvolvendo a competência de seus membros que, com o tempo, passam a ser especialistas e socializadores do conhecimento.

Três elementos são fundamentais para a caracterização de uma comunidade de prática: suas fronteiras;

Domínio - É o propósito compartilhado que orienta suas atividades e estabelece

Comunidade - São os membros, que ao desenvolverem atividades e discussões em conjunto, estabelecem relacionamentos e compartilham informações. Estas relações proporcionam aos participantes aprender uns com os outros e são essenciais para a caracterização da comunidade;

Prática - É um conjunto de esquemas de trabalho, ferramentas, ideias, estilos, linguagem, histórias e documentos decorrentes das interações na comunidade. A prática representa o conhecimento desenvolvido, compartilhado e armazenado, que se torna fonte de conhecimento para seus membros e outros que possam se beneficiar destes recursos.

Segundo Wenger (2000), para que as comunidades de prática sejam bem sucedidas é necessário que elas tenham a capacidade de serem sistemas de aprendizagem social. Healy (2009) define aprendizagem como o processo de evolução de um estado sem conhecimento para um onde há conhecimento. O aspecto social está nas interações humanas, aprender através do contato e associações regulares. A possibilidade de interagir e trocar experiências, fazendo com que os membros aprendam sobre um domínio e também sobre a própria participação, torna a comunidade de prática propícia a suportar a aprendizagem social (Ribeiro, 2011).

As relações e o sentimento de pertencer à comunidade são fundamentais no processo de aprendizagem social. A partir do momento que o participante adquire confiança e se identifica com seus pares estas relações se intensificam. Inicia-se um processo de motivação intrínseca, onde os participantes sentem-se a vontade para participar de discussões, expor suas ideias e socializar o conhecimento.

Em comunidades de prática a aprendizagem é construída de diferentes maneiras e o modo com que os participantes interagem define seu estilo. Cada um destes estilos tem associado a ele um conjunto de ferramentas que proporcionam suporte ao padrão de suas atividades.

Wenger, White e Smith (2010) sugerem diversas ferramentas para a instrumentalização em comunidades de prática, desde ferramentas de comunicação tradicionais como chat, fórum de discussão e e-mail até ferramentas mais sofisticadas 
como videoconferência e podcast ${ }^{2}$. Também são necessárias ferramentas de gestão e publicação. Sendo assim, a inovação não está nas ferramentas, mas sim no enfoque de seu uso, que permite aos participantes a disseminação do conhecimento (Silva, 2011).

As relações existentes entre as ferramentas, experiências e interesses pessoais fazem transparecer o domínio da comunidade de prática. Através delas é possível a inferência de interesses dos indivíduos para associá-los às comunidades, outros usuários ou temas que apresentam os mesmos interesses. Estas associações tem o objetivo de favorecer a disseminação do conhecimento, ampliar a abrangência das práticas e aumentar as relações, podendo recomendar novas e fortalecendo as existentes.

As ferramentas utilizadas em comunidades de prática proporcionam diferentes maneiras de o usuário interagir com o ambiente e, de todas estas, as que foram consideradas neste projeto são as de criação de conteúdo, onde a informação pode ser categorizada e seus temas relacionados aos interesses dos usuários.

\section{Sistemas de Recomendação}

Em um ambiente de comunidades de prática, onde as interações são incentivadas e a produção de conteúdo facilitada, a busca por informações específicas pode ser desgastante. Neste cenário os sistemas de recomendação são ferramentas essenciais, que orientam e auxiliam o usuário a encontrar conteúdo relevante.

Os sistemas de recomendação utilizam as preferências do usuário com o propósito de sugerir, de forma personalizada, novos itens de interesse. Eles são comuns em ambientes de comércio eletrônico e para o acesso a informação, sugerindo conteúdo, dentre um vasto espaço de possibilidades, que direcionam o usuário àqueles itens que melhor se enquadram em suas necessidades e preferências (Burke, 2002).

Diferentes técnicas são empregadas para produzir recomendações, dentre elas pode-se citar a filtragem colaborativa, a filtragem baseada em conteúdo e a filtragem híbrida (Cazella, 2010).

A filtragem colaborativa tem em sua essência a troca de experiências entre usuários que possuem interesses comuns (Cazella, 2010). Os itens são filtrados levandose em consideração avaliações feitas pelos usuários sobre um determinado assunto. A ideia básica é fazer com que participantes de determinada comunidade possam ser beneficiados pela experiência uns dos outros. Porém a maioria dos sistemas de filtragem colaborativa requer que os próprios usuários especifiquem o relacionamento de predição entre suas opiniões, ou indiquem os itens de interesse, ou indiquem pontuações dos itens pelos usuários (Herlocker, 2000). Um usuário de um sistema de filtragem colaborativa deve, portanto, pontuar cada item experimentado, indicando o quanto este item combina com sua necessidade de informação. A abordagem de filtragem colaborativa apresenta então uma média de pontuações para cada item com potencial de interesse.

Assim, o sistema indica ao usuário itens que são considerados de interesse pelo grupo e evita os itens de menor interesse. Neste tipo de filtragem é possível identificar nichos de interesse distantes através do reconhecimento de similaridades entre usuários e a compreensão ou reconhecimento do conteúdo não é necessário. Em geral, nos sistemas tradicionais de filtragem colaborativa, a recomendação baseia-se no histórico de avaliações comuns e no valor de predição calculado.

2 Arquivo de áudio digital (em geral em formato MP3) publicado na Internet e atualizado via RSS. 
Tipicamente o perfil do usuário em um sistema colaborativo consiste em um vetor de itens e suas avaliações, que cresce conforme as interações do indivíduo com o sistema.

A filtragem baseada em conteúdo consiste em categorizar e definir associações entre itens. O item a ser recomendado é aquele que apresenta o maior nível de similaridade com as escolhas prévias do indivíduo. Esta técnica é chamada de filtragem baseada em conteúdo porque o sistema realiza a filtragem baseada na análise de conteúdo do item e no perfil do usuário. A filtragem puramente baseada em conteúdo ignora a preferência de outros usuários.

Segundo Adomavicius (2005), ambas as filtragens apresentam limitações como o problema do novo usuário, quando o sistema não é capaz de gerar recomendações precisas para usuários que possuem poucas avaliações ou a super especialização, que ocorre quando o sistema recomenda apenas itens semelhantes a itens avaliados positivamente, não apresentando conteúdo diferente do perfil do usuário.

A fim de reduzir estas limitações a filtragem híbrida procura aprimorar suas recomendações, através da combinação de duas ou mais abordagens. É comum a filtragem colaborativa ou a filtragem baseada em conteúdo ser utilizada em conjunto com alguma outra para reduzir o problema do novo usuário, entretanto diversas combinações são possíveis (Burke, 2002). As técnicas de recomendação apresentam pontos fortes e fracos e a escolha de qual melhor se adapta depende do contexto de cada aplicação.

\section{Plataforma de Comunidades de Prática - CoPPLA}

A plataforma de comunidades de prática CoPPLA foi desenvolvida utilizando a solução tecnológica Python/Zope/Plone que disponibiliza um ambiente web para criação rápida e segura de sites. Os objetos publicados nas comunidades de prática são escritos em linguagem Python e armazenados em um banco de dados orientado a objetos nativo, denominado ZODB. Também escrito em Python, o ZODB persiste os objetos sem a necessidade de um mapeamento para tabelas de um banco relacional. A persistência dos objetos é transparente, tornando o código mais simples, robusto e legível (ZODB, 2013).

A indexação e pesquisa são responsabilidades do sistema de catálogo ZCatalog, que é composto por índices e metadados. Índices são campos que podem ser pesquisados e metadados são cópias de conteúdos dos objetos, que podem ser acessados sem a necessidade de acessar o próprio objeto (Plone, 2013).

$\mathrm{Na}$ plataforma CoPPLA os temas são palavras-chave representadas como entradas do índice Subject do ZCatalog. Um objeto pode ser marcado com um ou mais temas e, a partir destas palavras-chave, é possível pesquisar por conteúdos relacionados. Os usuários são objetos Python e, de forma direta, não existe relacionamento entre temas e usuários. Uma associação só é possível através dos itens publicados, que possuem um criador e podem ser marcados com temas, relacionando assim, os temas dos objetos a seus criadores. No entanto, essa abordagem torna difícil a execução de tarefas como a consulta por todos os temas relacionados a um usuário ou vice-versa. Também não abrange todos os interesses que um usuário pode apresentar, visto que o mesmo pode interessar-se por temas que não fazem parte de suas publicações. Devido a esta limitação, o objetivo deste artigo é apresentar um sistema de following planejado 
para proporcionar aos usuários novas formas de interação e a possibilidade de declarar, de forma explícita, suas preferências em comunidades de prática.

As relações entre usuários e temas são armazenadas utilizando-se o banco de dados objeto-relacional PostgreSQL. Bancos de dados relacionais persistem estas informações de forma mais direta e representativa do que o banco de dados orientado a objetos ZOBD. Além disso, PostgreSQL apresenta uma interface nativa para Python que possibilita a integração entre o banco de dados e a plataforma baseada em Python/Zope/Plone (Lerner, 2002).

Quando um usuário segue temas ou participantes é porque tem algum interesse nos mesmos. Estas informações, declaradas pelos usuários, servem de fontes para os sistemas de recomendação. Neste cenário, o sistema proposto foi validado através da implementação do sistema de following, ainda inexistente na plataforma CoPPLA, e de cenários para recomendação de outros usuários ou temas.

\section{Sistema de Following da CoPPLA}

O sistema de following proposto e desenvolvido para a plataforma CoPPLA foi concebido de forma a definir:

- O mecanismo de following entre usuários;

- O mecanismo de following entre usuários e temas;

- A medida de similaridade entre os usuários;

- Os usuários ou temas que serão recomendados, com base na rede de relacionamentos.

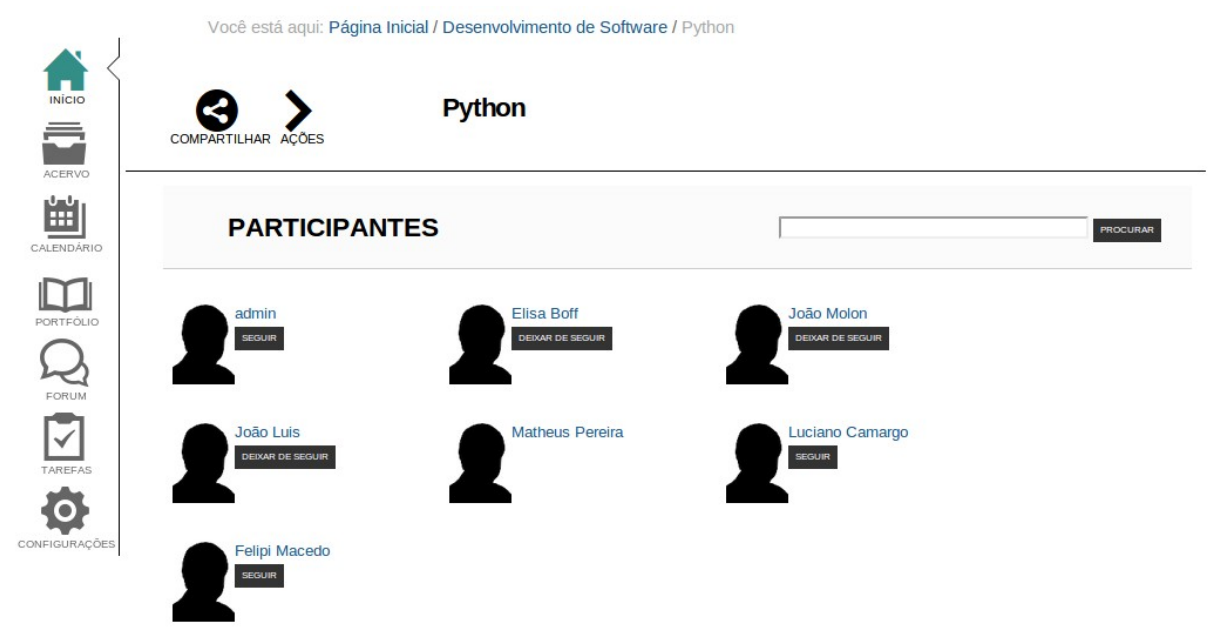

Figura 1 - Lista de Participantes de uma CoP

Atualmente, um dos espaços disponíveis nas comunidades de prática é a lista de participantes (Figura 1). Nela são apresentadas informações básicas de todos os usuários de uma comunidade. Nesta visualização, foi disponibilizado um mecanismo onde o 
usuário pode seguir outros participantes (following de usuários). Do mesmo modo, ao acessar os conteúdos de uma comunidade de prática, o usuário pode seguir os temas relacionados àquele conteúdo (following de temas), como representado na Figura 2.

O sistema de following armazena estas relações através do PostgreSQL e a conexão entre a plataforma e o banco de dados acontece através do SQLAlchemy, um conjunto de ferramentas e ORM (Object Relational Mapper) que faz o mapeamento das definições de classes Python em tabelas de bancos relacionais e dos objetos em entradas de tabelas (SQLAlchemy, 2013). São representados no banco de dados os usuários, os temas, as relações entre usuários e as relações entre usuários e temas.

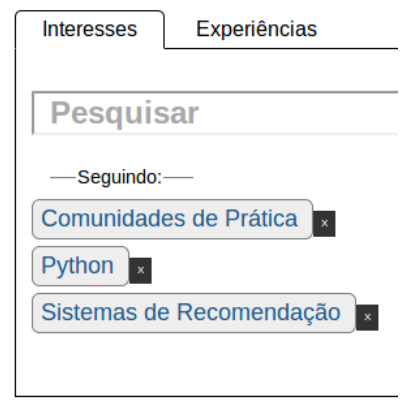

Figura 2 - Lista de Temas que um usuário segue

Ao seguir temas e usuários o participante de uma CoP relaciona seus interesses aos de outros membros desta rede de relacionamentos. Indiretamente, ao seguir outros usuários, o indivíduo coloca este conteúdo a disposição de forma implícita. Um sistema de following pretende capturar todas estas relações, gerenciando os interesses que estão distribuídos em uma vasta rede de relacionamentos, sem a necessidade de uma formalização substancial de conteúdos avaliados por uma grande quantidade de usuários, requisitados para um sistema de recomendação. Levando em consideração que o requisito básico da plataforma CoPPLA são as redes de relacionamento e os temas que conectam estas redes, algumas características são importantes para prover as recomendações: as CoP focam principalmente nas trocas entre os pares e em quanto a necessidade de interação sobre determinado tema é inversamente proporcional ao tempo de aprendizagem em sua compreensão e aplicação; a recomendação de conteúdos diferentes dos já avaliados é importante na medida que novos temas emergem das interações dentro das comunidades, e o conhecimento de domínio está implícito na comunidade não sendo necessário considerá-lo em uma matriz de cálculos para recomendações.

A partir desta análise é possível identificar a técnica de filtragem colaborativa como sendo a que melhor se enquadra no contexto da aplicação de following de comunidades de prática, proposta neste artigo. Através dela é possível gerar recomendações cruzadas, podendo sugerir itens completamente diferentes dos já avaliados, identificando usuários com preferências similares, declaradas explicitamente através do following, e indicando itens ainda não avaliados uns aos outros. Nesta técnica também não é necessário o reconhecimento do conteúdo a ser recomendado.

O perfil do usuário é representado por um grafo proveniente das relações do following, assim como na teoria sociométrica de Moreno (1992), tanto de usuários quanto de temas, e é utilizado como entrada para o cálculo de similaridade no processo 
de recomendação. Esta representação, dos interesses do usuário através do following, é importante a medida em que reduz o esforço computacional para a inferência dos interesses dos participantes.

Três métricas são utilizadas para o cálculo de similaridade entre os usuários: cosseno do following de usuários, cosseno do following de temas e força do relacionamento. A força do relacionamento entre dois usuários leva em consideração a distância necessária para alcançar determinado usuário, a partir de uma busca em amplitude no grafo, e todas as relações de following encontradas durante este percurso.

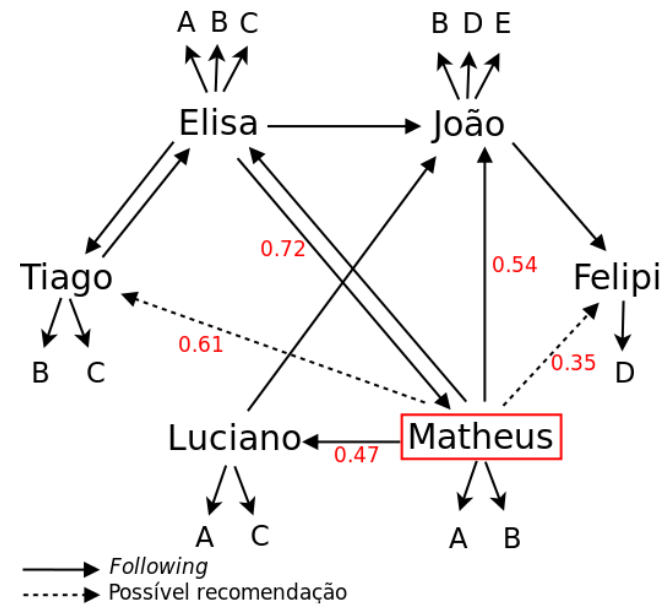

Figura 3 - Similaridade calculada para Matheus

Em um ambiente de comunidades de prática com um conjunto de usuários $\mathbf{U}=$ \{Elisa, Felipi, João, Luciano, Matheus, Tiago $\}$ e um conjunto de temas $\mathbf{T}=\{\mathbf{A}, \mathbf{B}, \mathbf{C}$, D, E\}, relacionados através do sistema de following, pode-se sugerir novas ligações calculando a similaridade entre os usuários da rede, indicando aqueles com maior similaridade que ainda não possuem ligação direta. Por exemplo, para o usuário alvo Matheus, o usuário recomendado será Tiago, seguido pela recomendação de Felipi, como demonstrado na Figura 3 e na Tabela 1.

Tabela 1 - Similaridade entre o usuário alvo e demais usuários

\begin{tabular}{|c|c|c|c|c|c|}
\hline $\begin{array}{l}\text { target_member } \\
\text { [PK] character var [ }\end{array}$ & $\begin{array}{l}\text { recommended_member } \\
\text { [PK] character varying }\end{array}$ & $\begin{array}{l}\text { cosine_tags } \\
\text { double precision }\end{array}$ & $\begin{array}{l}\text { cosine_users } \\
\text { double precision }\end{array}$ & $\begin{array}{l}\text { bfs_graph } \\
\text { double precision }\end{array}$ & $\begin{array}{l}\text { similarity } \\
\text { double precision }\end{array}$ \\
\hline \begin{tabular}{l|l} 
matheus & $\epsilon$
\end{tabular} & elisa & 0.816496580927726 & 0.333333333333333 & 1 & 0.716609971420353 \\
\hline matheus & felipi & $\theta$ & 0.577350269189626 & 0.472222222222222 & 0.349857497137283 \\
\hline matheus & joão & 0.408248290463863 & 0.577350269189626 & 0.625 & 0.536866186551163 \\
\hline matheus & luciano & 0.5 & 0.408248290463863 & 0.5 & 0.469416096821288 \\
\hline matheus & matheus & 1 & 1 & 1 & 1 \\
\hline matheus & tiago & 0.5 & 0.577350269189626 & 0.75 & 0.609116756396542 \\
\hline
\end{tabular}

A última coluna da Tabela 1, "similarity double precision" indica o valor calculado pelo algoritmo de recomendação. O valor igual a 1 representa o próprio usuário. O algoritmo, ao montar a lista de sugestões, ignora a recomendação de usuários que o membro da comunidade já segue, como é o caso do usuário Elisa, que o usuário 
Matheus já segue. Por isso, com os valores ordenados de forma decrescente, as próximas sugestões do algoritmo serão os usuários Tiago (s=0.60911), e Felipi ( $\mathrm{s}=0.34985)$, nesta ordem.

\section{Considerações Finais}

O presente artigo abordou a importância dos relacionamentos entre os usuários no contexto de comunidades de prática e de que forma a utilização de ferramentas de comunicação colabora com o desenvolvimento social do conhecimento.

Os sistemas de recomendação utilizam técnicas para entregar conteúdo personalizado a um usuário, considerados mais relevantes ao perfil deste usuário, facilitando o acesso à informação e convidando-o a experimentar novos conhecimentos.

Para que um sistema de recomendação possa gerar sugestões de qualidade, ele deve manter um modelo de usuário de forma a representar seus interesses. Foram apresentadas três técnicas de recomendação e, ao considerar as características das comunidades de prática, definiu-se a filtragem colaborativa como a técnica de sistemas de recomendação a ser utilizada neste projeto. O sistema de following gera um grafo direcionado de interações, identificando a importância e a força entre as relações. Com base nestas relações, extraídas da filtragem colaborativa, o sistema apresenta a um membro de uma comunidade sugestões de usuários com interesses comuns e possíveis temas de interesse.

O desenvolvimento do sistema de following agregou novas funcionalidades à plataforma de comunidades de prática, CoPPLA, proposta inicialmente em Fiorio (2011) e Ribeiro (2011). Estas funcionalidades ampliam e estimulam as interações entre os participantes das comunidades de prática e colaboram com o processo de aprendizagem. Os sistemas de recomendações ainda proporcionam um ambiente com conteúdo personalizado que garante uma participação de diferentes níveis e mantém ativo o diálogo e práticas nas comunidades.

\section{Referências}

Adomavicius, G. and Tuzhilin, A. Toward the next generation of recommender systems: a survey of the state-of-the-art and possible extensions. Knowledge and Data Engineering, IEEE Transactions on, 17(6):734-749, Junho 2005.

Burke, R. Hybrid recommender systems: Survey and experiments. User Modeling and User-Adapted Interaction, 12(4):331-370, 2002.

Cazella, S. C., Nunes, M. A. S. N. and Reategui, E. A. A ciência da opinião: estado da arte em sistemas de recomendação. 1:161-216, 2010. André Ponce de Leon F. de Carvalho; Tomasz Kowaltowski..(Org.). Jornada de Atualização de Informática-JAI 2010-CSBC2010. Rio de Janeiro: PucRIO.

CoPPLA - Communities of Practice Plataform. Technical report, 2013. Disponível em: <http://www.communitas.org.br/communitas/products>. Acessado em 14/05/2013.

Fiorio, M., Silva, J. L. T. and Ribeiro, A. M. Um framework de comunidades de prática 
em ambientes virtuais de aprendizagem. RENOTE - Revista Novas Tecnologias na Educação, 2011.

Healy. A. Communities of practice as a support function for social learning in distance learning programs. M.D. Lytras, al. et (Eds.), WSKS 2009, CCIS 49, pages 49-56, 2009.

Herlocker, J. L. Understanding and Improving Automated Collaborative Filtering Systems. PhD thesis, University of Minnesota, September 2000.

Lerner, R. M. Databases and zope. linuxjournal, Maio 2002. Disponível em: <http://www.linuxjournal.com/article/5849>. Acessado em 16/06/2013.

Moreno, J. L. Quem Sobreviverá: Fundamentos da Sociometria, Psicoterapia de Grupo e Sociodrama. Volume 1. Goiânia: Dimensão, 1992.

Plone - catalog. Technical report, Plone.org, 2013. Disponível em: $<$ http://developer.plone.org/searching and indexing/catalog.html>. Acessado em 20/06/2013.

Ribeiro, A. M., Silva, J. L. T., Boff, E. and Viccari, R. M. Dos ambientes de aprendizagem às comunidades de prática. Anais do XXII SBIE - XVII WIE, 2011. Aracaju, 21 a 25 de novembro de 2011.

Silva, J. L. T., Ribeiro, A. M. and Fiorio, M. Um estudo de implantação de comunidades de prática em um portal institucional. RECIIS - R. Eletr. de Com. Inf. Inov. Saúde, 3(3), 2011. Rio de Janeiro.

SQLAlchemy - Object Relational Tutorial. Technical Report, 2013. Disponível em: $<$ http://docs.sqlalchemy.org/en/rel 0 8/orm/tutorial.html>. Acessado em 20/06/2013.

Wenger, E. Communities of practice: Learning, meaning, and identity (Learning in Doing: Social, Cognitive and Computational Perspectives). Cambridge University Press, 1998. ISBN 978-0-521-66363-2.

Wenger, E. Communities of practice and social learning systems. 7(2):225-246, Maio 2000. Wenger Organization.

Wenger, E., White, N. and Smith, J. D. Digital Habitats: stewarding technology for communities. CPsquare, 2010.

Zaiane, O. R. Web mining: Concepts, practices and research. Simpósio Brasileiro de Banco de Dados, SBBD, 43(8):410-474, 2000. João Pessoa.

ZODB - A Native Object Database for Python. Technical Report, 2013. Disponível em: $<$ http://zodb.org/>. Acessado em 07/06/2013. 